\title{
Linx
}

Revue des linguistes de l'université Paris X Nanterre

$73 \mid 2016$

Énonciation et marques d'oralité dans l'évolution du français

\section{Perception visuelle, inférence et polyphonie : de il pert que à il paraît que}

\section{Amalia Rodríguez Somolinos}

\section{(2) OpenEdition}

\section{Journals}

Édition électronique

URL : http://journals.openedition.org/linx/1623

DOI : $10.4000 /$ linx. 1623

ISSN : 2118-9692

Éditeur

Presses universitaires de Paris Nanterre

Édition imprimée

Date de publication : 1 septembre 2016

Pagination : 17-38

ISSN : 0246-8743

Référence électronique

Amalia Rodríguez Somolinos, « Perception visuelle, inférence et polyphonie : de il pert que à il parait que », Linx [En ligne], 73 | 2016, mis en ligne le 01 mars 2017, consulté le 19 avril 2019. URL : http:// journals.openedition.org/linx/1623; DOI : 10.4000/linx.1623 


\title{
Perception visuelle, inférence et polyphonie : de il pert que à il paraît que
}

\author{
Résumé $^{1}$
}

Nous étudions ici l'évolution sémantique de la structure impersonnelle il paraît que p depuis l'ancien français il pert que jusqu'à aujourd'hui. Cette évolution s'est faite en trois étapes:

1. Jusqu'au XIV ${ }^{\mathrm{e}}$ siècle, il pert que peut relever de la perception visuelle directe. En français médiéval et préclassique, il pert que, il appert que, il paroist que ont le plus souvent une valeur médiative inférentielle, avec un sens « on voit bien que, il est évident que ». En français classique il paraît que1 vient remplacer il paroist que avec la même valeur sémantique. Le lien inférentiel mis en place par ces marqueurs se présente comme intersubjectif. Il relève du savoir en général.

2. En français classique se développe il paraît que2, qui a évolué vers une plus grande subjectivité. Le marqueur indique que le locuteur a des raisons pour induire $\mathrm{p}$ à partir de certains indices, qui ne sont pas toujours repérables.

3. Il paraît que3 est un marqueur d'ouï-dire. Il devient le seul emploi possible à partir de 1925 environ. Il paraît que effectue une mise à distance; le locuteur ne prend pas entièrement en charge l'assertion sur laquelle porte le marqueur.

Il paraît que $\mathrm{p}$ a connu ainsi un affaiblissement en diachronie. Il a évolué vers une plus grande subjectivité et un degré moindre de certitude de la part du locuteur.

Abstract

We study here the semantic evolution of the impersonal structure Il paraît que $\mathrm{p}$ from Old French il pert que until nowadays. This evolution has followed three stages:

1. Until the $14^{\text {th }}$ century, il pert que is linked with direct visual perception. In Medieval and Preclassical French, il pert que, il appert que, il paroist que have most often an inferential evidential use with a meaning "it is clear that, it is obvious that". in Classical French il paraît que1 replaces il paroist que with the same meaning. All three markers make possible an intersubjective reading, which has to do with knowledge in general.

2. In Classical French appears il paraît que2, which has evolved towards greater subjectivity. The markers indicates that the speaker has reasons to infer $\mathrm{p}$ from a number of clues, which are difficult to determine.

3. Il paraît que3 is a hearsay marker. This is the only possible use from 1925 onwards. With il paraît que3, the speaker takes some distance with respect to the evidence. He does not commit himself entirely to the assertion.

Il paraît que has seen its modal value weaken over time. It has evolved towards greater subjectivity and a lower degree of certitude from the speaker.

1 Cet article a été réalisé dans le cadre du projet de recherche FFi2013-41355-P « Marqueurs pragmatiques et oralité en linguistique historique du français » du Ministerio de Economía y Competitividad, Espagne, (Plan Estatal i+D+i 2013-16) 


\section{INTRODUCTION}

Nous étudions ici l'évolution de il paraît que p depuis l'ancien français il pert que/ il apert que jusqu'à aujourd'hui. Dans la structure impersonnelle moderne il paraît que p, le verbe paraître est au présent de l'indicatif et $\mathrm{p}$ est une proposition subordonnée. Cette étude se situe dans le cadre de la sémantique pragmatique. Nous utiliserons plus spécialement les notions de médiativité (angl. evidentiality), de modalité et de polyphonie. La valeur modale, comme on le sait, a trait à l'attitude du locuteur vis-à-vis de ce qu'il dit. La valeur médiative est en rapport avec la nature de la source, le marqueur indique comment le locuteur obtient ou crée l'information.

En français médiéval, les verbes paroir (var. pareir, parair), aparoir (var. apparoir), très proches étymologiquement et sémantiquement, présentent tous les deux une construction impersonnelle il pert que, il apert que signifiant « on voit bien que, il est visible/ évident que ». De façon parallèle, la langue connaît la structure en incise correspondante si comme il pert. Comme le signale le Dictionnaire de Huguet, la construction impersonnelle peut présenter en moyen français un verbe au présent de l'indicatif, mais elle existe aussi avec le même sens avec le verbe au futur (il apperra) et au présent du subjonctif (qu'il appere).

À côté du verbe paroir (lat. parere), l'ancien et le moyen français connaissent un verbe pareistre, paroistre issu du latin tardif parescere, qui est beaucoup moins fréquent (voir Lanly 1971 et Rey 2004). Le verbe paroir disparait à la fin du $x v i^{\mathrm{e}}$ siècle remplacé par pareistre, paroistre.

En français classique (1650-1789), paroistre coexiste avec la forme paraître, qui finira par s'imposer à partir du XVIII ${ }^{\mathrm{e}}$ siècle. Il paroist que, caractéristique du français préclassique (1550-1650), sera remplacé par il paraît que, dont la première occurrence dans Frantext date de 1655. Ces variations morphologiques et graphiques n'ont pas d'incidence sur le fonctionnement sémantique de la construction impersonnelle.

Dès l'ancien français, il pert que a un rapport étroit avec la perception visuelle. Le locuteur est un témoin direct et réalise une constatation. Les indices perceptifs visuels sont considérés au Moyen Age comme une garantie de vérité, comme une source digne de foi et même comme une preuve admissible en justice. C'est la raison pour laquelle les marqueurs médiatifs perceptifs sont particulièrement fréquents en ancien français, alors qu'en français moderne ils sont beaucoup moins usuels. Ce même phénomène est signalé pour l'anglais par Chafe (1986:268).

Le verbe voir, comme on le sait, est polysémique. Il peut signifier voir physiquement, avec les yeux, et aussi comprendre " je vois ce que vous voulez dire ». Grossman et Tutin (2010) parlent d'un sens perceptif et d'un sens intellectuel. Les deux sens sont anciens, d'après Rey (2004) ils existaient déjà en indo-européen et en latin. Certains marqueurs présentent le même glissement du concret vers l'abstrait. C'est le cas par exemple de estre avis, il y a d'une part une activité physique concrète liée à la perception visuelle, d'autre part une opinion, un jugement, une activité mentale plus proche de croire, penser ou encore de devoir épistémique (voir Rodríguez Somolinos, 2014). Beaucoup de marqueurs ayant trait à la perception visuelle présentent également une valeur médiative inférentielle : c'est le cas de apparemment, de il m'est avis que, ainsi que du français médiéval il pert que et de l'emploi ancien de il paraît que, qui a été en usage jusqu'au début du $\mathrm{xx}^{\mathrm{e}}$ siècle.

Dans l'usage actuel, il paraît que est un marqueur d'ouï-dire. Dans Il paraît que Jean est malade, le marqueur renvoie à un dire, à une information rapportée. La source de l'information se trouve soit 
dans des propos d'autrui, soit dans des rumeurs. Nous verrons ainsi comment un marqueur relevant au départ de la perception visuelle a pu évoluer depuis une valeur médiative inférentielle jusqu'à une valeur d'ouï-dire.

Pour le français médiéval, notre corpus provient de la Base du français médiéval (BFM), de la base Textes de Français Ancien (TFA), ainsi que du Corpus de la littérature médiévale. Pour les textes postérieurs à 1500, nous avons utilisé la base Frantext, complétée par des dépouillements personnels.

\section{PERCEPTION VISUELLE ET INFERENCE : DE L'ANCIEN FRANÇAIS AU FRANÇAIS CLASSIQUE}

\subsection{Ancien français : il pert que, il apert que, il appert que}

En ancien français, la structure impersonnelle il pert que, avec le verbe paroir au présent de l'indicatif, relève de la perception visuelle, qu'il s'agisse de perception physique ou intellectuelle. Il apert que, du verbe aparoir, est également attesté avec le même sens dès l'ancien français. Peu fréquent au départ, sa fréquence augmente en moyen français. Ces deux marqueurs, avec un sens « on voit bien que, il est visible que », peuvent renvoyer à une expérience visuelle directe du locuteur :

(1) Et Jehanz, au plus tost qu'il pot,

A la sepolture reclose,

Si qu'il ne pert a nule chose

Que l'an i eüst atochié.

(Chrétien de Troyes, Cligès, p. $187 ; 1176$ )

[Et, le plus vite possible, Jean a refermé le tombeau, de telle sorte qu'on ne voit aucunement qu'on y ait touché.]

En (2), le marqueur apparaît dans une construction en incise si com il apert encore avec une valeur qui est aussi purement visuelle :

(2) ... mes maintenant entra laienz une lance, dont il fu feruz parmi oultre les deus cuisses, si durement qu'il en remest mehaigniez si com il apert encore, ne onques puis n'en pot garir, ne ne fera devant que vos vendroiz a lui. (La Queste del Saint Graal, p. 209 ; 1225)

[mais alors entra là-dedans une lance, qui traversa ses deux cuisses, si fortement qu'il en est resté blessé, comme on peut encore le voir, et il n'a jamais pu guérir, et il ne le pourra pas si vous ne venez pas à lui.]

Il est rare cependant que il pert/ apert que soit purement visuel. Il réalise souvent une inférence basée sur une perception visuelle, comme en (3) et (4) :

(3) - Voirs est, fet mes sire Gauvains ;

dame, je sui trestoz certains

que de rien nule ne mantez :

ses haubers est ansanglantez,

molt est hurtez et debatuz ;

bien pert que il s'est conbatuz;

savoir poons, sanz nule faille,

que forz a esté la bataille.

(Chrétien de Troyes. Erec et Enide, p. 36; 1170) 
[Son haubert est ensanglanté, il est tout meurtri et contusionné; on voit bien qu'il a combattu; nous pouvons savoir sans aucun doute que la bataille a été dure.]

En (3) Gauvain voit le haubert ensanglanté et les meurtrissures du chevalier, ce sont là des indices visibles qui lui permettent de déduire qu'il a combattu.

(4) Le mescinete va tramblant, car il pert bien a son samblant qu'ele a la fors tel cose oïe dont gaires ne s'est esjoïe ; (Gautier d'Arras, Eracle, p. 81 ; 1176-1184)

[La jeune fille tremble, car on voit bien à son semblant qu'elle a entendu là dehors quelque chose qui ne lui a pas fait plaisir.]

Remarquons qu'en ancien et en moyen français la structure impersonnelle est syntaxiquement libre. Le verbe se conjugue à l'imparfait, (il) paroit que, ou au passé simple, (il) parut que, le sens étant le même :

(5) Au roi Phenis l'ont presentee.

Li rois l'a souvent esgardee ;

il paroit bien a son visage que

ele estoit de haut parage. (Floire et Blancheflor, v. 1.107 ; c. 1150-1160)

[ils l'ont offerte au roi Félis. Le roi l'a regardée longuement; on voyait bien à son visage qu'elle était de haute naissance.]

(6) Chevalier fu de grant corage,

Mainte plaie out en son visage ;

Hardiz esteit, assez parut

As granz plaies que il reçut. (Wace, Roman de Rou, 1. 11.155, vol. 2 ; c. 1170)

[C'était un chevalier de grand courage, il avait beaucoup de blessures au visage ; il était audacieux, cela se voyait bien aux grandes blessures qu'il avait reçues.]

De la même façon il pert que, peut être nié sans que son sens soit modifié. Cela donne une structure mal pert que $p$, qui est attestée en ancien et en moyen français:

(7) Pour Dieu, dont venés vous? Ne me chelés noient !

Vous avés bien pensé de vous, chertainement, car poi estes hallés de vis [et] de jouvent :

mal pert a vo viaire que n'aiés point d'argent.

(Beaudouin de Sebourc, p. 156; 1350)

Mal pert que p signifie «il est évident que non-p». Il faut comprendre ici «En vous voyant, on ne peut pas dire que vous n'ayez pas d'argent », c'est-à-dire «il est évident à votre aspect que vous avez de l'argent».

2.2. Moyen français : il pert que, il appert que marqueurs inférentiels

En moyen français, il pert que présente des emplois similaires à ceux qu'il avait en ancien français. Il peut avoir un sens perceptif intellectuel :

(8) L'Abbesse : Messire Nicole, par foy,

Vous soiez li tresbien venuz.

Il pert bien qu'estes devenuz 
Un grant sire ; on ne vous voit mais.

(Miracle de l'abbeesse grosse, p. 82, vol. 2 ; c. 1340)

[On voit bien que vous êtes devenu un grand seigneur, on ne vous voit plus.]

Il s'agit ici d'une inférence par raisonnement sans rapport avec la perception visuelle directe. À partir d'un argument on ne vous voit mais, l'abbesse conclut à «vous êtes devenu un grand seigneur ».

Il pert que, du verbe paroir, avec le sens « on voit bien que, il est évident que » est d'un usage courant en ancien français et au début du moyen français. Dans le courant du XIV ${ }^{\mathrm{e}}$ siècle, il est remplacé par il apert/appert que, du verbe aparoir/ apparoir. Il appert que apparait souvent en moyen français dans des structures de type, et par ce appert que, et pour ce il appert que, par quoy il appert que, dans lesquelles l'inférence est explicitée. Elles sont souvent employées dans des textes argumentatifs :

(9) ... car a ce propos racompte saint Jeroisme en son livre contre Jovinian, comment Platon ellut pour demourer une ville champestre nommee Achademie, laquele estoit loing de Athenes et de toutes autres citz, a celle fin qu'il peust luxure eschever et vivre chastement. Et Tertulian recite comment Democrite se creva les yeulx affin qu'il ne veist les femmes, car il ne les pouoit regarder sanz pechi. Par quoy il appert qu'il ne souffist mye chastet avoir, mais aussi doit on les regars eschever. (Jacques Legrand, Livre de bonnes meurs, p. 326, Partie i, Ch. XII; 1410)

La structure par quoy il appert que p signale explicitement sa fonction inférentielle. Le locuteur s'appuie sur un certain nombre d'arguments pour conclure à p, qui est pris en charge avec un degré élevé de certitude. La construction est traduisible par « ce qui vient prouver que ». Elle admet une paraphrase " à partir des arguments ci-dessus, il apparaît nettement que » ${ }^{2}$ L'enchaînement argumentatif ne dépend pas de la subjectivité du locuteur ; il est présenté comme quelque chose d'évident, qui ne peut pas être mis en doute. Le Dictionnaire de la langue française du XVI siècle, de Huguet, donne comme traduction pour il appert impersonnel «il apparaît, il est visible, évident, certain ». Il pert que et il apert que sont des marqueurs médiatifs. On peut les classer, en fonction de la source de l'information, d'une part dans la catégorie de l'expérience directe visuelle, avec les verbes de perception comme veoir, esgarder, d'autre part dans la catégorie de l'expérience indirecte par inférence (voir Chafe, 1986 : 266-267 et Dendale Tasmowski $2001: 343$ ).

\subsection{Français préclassique: il appert que, il paroist que}

La construction il paroist que, il paroist bien/ assez que, du verbe paroistre, apparaît dans le dernier quart $\mathrm{du} \mathrm{xvi}^{\mathrm{e}}$ siècle, en concurrence avec il appert que qu'elle finira par remplacer. En français préclassique (1550-1650), le marqueur correspond dans tous les cas aux emplois médiévaux : inférence basée sur une perception visuelle (10), ou inférence par raisonnement « on voit bien que, il est évident que » (11).

(10) Mélibée : Tu es [es] bien vieille. On dit bien vray, qu'en vain ne cheminent les jours. Si Dieu me gard, je t'avois mescogneuë, n'estoit cette petite marque que tu as au visage. Il paroist bien

\footnotetext{
2 L'étude de la construction impersonnelle il apparaît que reste à faire. Le français préclassique (1550-1650) connaît il aparoist que, du verbe aparoistre, le plus souvent dans des suites de type d'où/ par où il apparoist que. Cette construction est très peu fréquente; elle ne présente que 6 occurrences dans Frantext. Le français classique connait, par où il paroît que, par où il paraît que, par où il est évident que. La construction moderne il apparaît que ne date que de la fin du XIX ${ }^{\mathrm{e}}$ siècle. Le premier exemple dans Frantext est de 1893.
} 
que tu as esté belle, tu me sembles à cette heure toute autre : Tu t'[es] fort changee. (Jacques de Lavardin, La Célestine, p. 96 ; 1578)

(11) Lucrèce : Bon prou vous face ma tante, et la noble compagnie. Dieu benie tant de gens de bien, et si honorables.

Célestine : Tant de gens, ma fille ? Te semble-il qu'ils soient beaucoup ? Il paroist bien que tu ne m'as cogneuë en ma prosperité. Aujourd'huy a vingt ans que qui me vit, et maintenant me voit, je m'estonne que le cueur ne luy creve de douleur. (Jacques de Lavardin, La Célestine, p. 160 ; 1578)

En (11) Célestine utilise les propos de Lucrèce comme un indice à partir duquel elle conclut à « tu ne m'as pas connue dans ma prospérité ».

Comme en moyen français (voir 9), l'inférence par raisonnement est souvent réalisée par des structures qui explicitent la structure logique : par où il appert que, de tout cecy appert que, d'où il appert que, parquoy il appert que, «d'où l'on peut déduire que / ce qui vient prouver que »:

(12) [Le diable] séduit en erreur les esprits des hommes, d'autre part il suscite haine et enflambe contentions et noises, le tout afin de renverser le règne de Dieu et de plonger les hommes en damnation éternelle. Dont il appert que de nature il est pervers, meschant et malin. (Jean Calvin, Institution de la religion chrestienne. Livre i, p. 197-198 ; 1560)

Jean Calvin, dans un texte argumentatif, fait un large usage de dont il appert que/ de là il appert que, etc. pour appuyer son argumentation. En (12), le locuteur donne d'abord plusieurs arguments, sur lesquels il va s'appuyer au moyen de dont il appert que pour présenter une conclusion. D'un point de vue modal, le locuteur se montre certain de ce qu'il asserte.

Dans cette première étape de l'évolution, il pert que/ il appert que/ il paroist que présentent une valeur intersubjective (voir Nuyts 2001). Ils correspondent à ce que Traugott (1989 : 43-47) appelle a weak subjective epistemic. Ils concernent le savoir en général, comme le signale Hanson (1987 : 136) : «[It] seems to suggest that anyone would be expected to subscribe to the same beliefs ». L'enchaînement argumentatif mis en place par dont il appert que se présente comme indépendant de la subjectivité du locuteur, il s'agit d'une inférence que tout le monde pourrait établir. 


\subsection{Français classique: il paroist que, il paraît que 1}

En français classique (1650-1789), le verbe paraître vient concurrencer paroistre, qu'il finira par remplacer au début du XVIII ${ }^{\mathrm{e}}$ siècle. Vers 1650 apparaît la construction moderne il paraît que, dont le fonctionnement sémantique est le même que celui de il paroist que :

(13) Je reçois deux de vos lettres, ma chère enfant, mais mon Dieu, que la première m'aurait donné de violentes inquiétudes si je l'avais reçue sans la seconde, où il paraît que la fièvre de ce pauvre chevalier s'est relâchée et lui a donné un jour de repos ! (Mme de Sévigné, Correspondance, t.3, 1680-1696, p.721; 1689)

La lettre indique clairement que " la fièvre de ce pauvre chevalier s'est relâchée et lui a donné un jour de repos ». Etant donnée la fiabilité de la source de l'information, il paraît que doit être interprété comme « on voit bien que » et non pas comme « il semble que », plus subjectif, qui correspondrait à la deuxième étape dans l'évolution du marqueur. L'exemple (14) est à rapprocher de (9) et de (12) cidessus :

(14) Les fouilles que l'on a faites en d'autres circonstances pour former des citernes, ont fait découvrir des ruines souterraines, d'après lesquelles il paraît que la ville moderne est bâtie sur l'ancienne. (Comte de Volney, Voyage en Égypte et en Syrie, p. 171, chap. 29 ; 1787)

Nous appellerons ce premier emploi il paraît que1. Il est proche de il pert que, de il appert que et de il paroist que. Il paraît que1 peut avoir deux sens différents en fonction du contexte, soit « on voit bien que, il est évident que », soit « d'où l'on peut déduire que / ce qui vient prouver que ». Il est en usage en français classique jusqu'au XVIII ${ }^{\mathrm{e}}$ siècle.

\section{INFERENCE ET SUBJECTIVITE :}

\section{IL PARAIT QUE2}

À partir du français classique et jusqu'au début $d u x x^{\mathrm{e}}$ siècle, il paraît que évolue vers une plus grande subjectivité. Il suit en cela une tendance générale dans l'évolution des marqueurs épistémiques : «Meanings tend to become increasingly based in the speaker's subjective belief state/ attitude towards the proposition » (Traugott, 1989 : 35). Dans cette deuxième étape, il paraît que est en rapport avec le savoir du locuteur en particulier. Il correspond à ce que Traugott (1989 : 41) appelle a strongly subjective epistemic meaning. Il paraît que acquiert ainsi une valeur inférentielle différente. Il réalise une inférence par raisonnement, basée sur les connaissances du locuteur ou sur des indices qui ne sont pas toujours repérables. Nous parlerons alors de il paraît que2 :

(15) Vous me paraissez bien contente du bon prélat ; j'aime à vous voir ces sentiments. Vous y mêlez même la crainte qu'on a toujours avec les vieilles gens, c'est de croire qu'ils vous vont échapper ; je crains cette perte pour le moins autant que vous. Il paraît que vous n'aviez que lui pour secrétaire, car vous avez fait toutes vos écritures vous-même. (Mme de Sévigné, Correspondance, t. 2, p. $900 ; 1680$ )

(16) Le père Receveur qui y descendit, nous rapporta que ce marais était bordé des plus belles plantations de bananiers et de mûriers. Il paraît, comme nous l'avions observé en naviguant le long de la côte, qu'il s'est fait un éboulement considérable vers la mer, qui a occasionné une grande brèche à ce cratère (Voyage de La Pérouse autour du monde, Ch. V, t.2, p. 101; 1797) 
Dans cet emploi, il paraît que2 inférentiel est à rapprocher du apparemment et du il semble que du français moderne. En (16) la brèche visible dans le cratère permet d'établir par inférence qu'il y a eu un éboulement vers la mer. Il s'agit donc d'une inférence à partir d'une perception visuelle. Le marqueur n'a pas besoin d'un contexte explicite marquant l'inférence, ce qui signifie que l'élément inférentiel fait maintenant partie du sens de il paraît que2. Le critère principal pour distinguer les deux premiers emplois de il paraît que est la traduction en français moderne. Il paraît que1 signifie « on voit bien que, il est évident que », alors que il paraît que2 équivaut à « apparemment, il semble que ». Ce dernier est plus subjectif et présente un degré moindre de certitude.

On peut appliquer à il paraît que2 la description sémantique que donne Nølke (2001 : 21) pour il semble que en français moderne : «Il semble que sert à diluer la responsabilité inhérente à l'acte de parole. [..] En énonçant il semble que p, le locuteur présente l'existence du point de vue véhiculé par $\mathrm{p}$ comme étant une conclusion qu'il tire à partir d'un certain nombre de signes ou d'indices plus ou moins inconscients ». Il semble que est un marqueur inférentiel, mais ces inférences se basent sur des indices présentés comme non repérables. Voilà pourquoi cette construction introduit toujours une nuance de subjectivité.

Il paraît que 2 marque une mise à distance ; l'assertion p est prise faiblement en charge par le locuteur. Celui-ci ne se rend pas entièrement responsable de la vérité de $p$, il se limite à indiquer qu'il a des raisons pour induire $\mathrm{p}$ à partir de certains indices, qui peuvent même être inconscients. Comme apparemment, il paraît que2 est un atténuateur, il comporte souvent, à l'instar de il semble que, une nuance de doute ou d'incertitude :

(17) L'accusé n'ayant point comparu, les pairs de France le condamnèrent à mort, et déclarèrent toutes ses terres situées en France acquises et confisquées au roi. Philippe se mit bientôt en devoir de recueillir le fruit du crime du roi son vassal. Il paraît que le roi Jean était du naturel des rois tyrans et lâches. Il se laissa prendre la Normandie, la Guyenne, le Poitou, et se retira en Angleterre, où il était haï et méprisé. (Voltaire, Essay sur l'histoire générale ..., chap. 40 , p. $309 ; 1756$ )

En (17), Voltaire ne peut pas être entièrement sûr de ce qu'il asserte. Il utilise il paraît que pour modaliser la prise en charge de p, avec un sens « il semble que, tout semble indiquer que ».

Il paraît que2 est multifonctionnel, il présente des emplois qui varient en fonction du contexte ${ }^{3}$. Toujours inférentiel, la source de l'information, ainsi que le degré de certitude du locuteur peuvent varier. Il s'agit parfois d'une conjecture, une supposition faite par le locuteur à partir d'indices difficiles à déterminer. Cela est visible dans la combinaison de il paraît que2 avec vraisemblablement en (18) :

(18) J'aspirais au moment d'être libre et d'échapper à mes ennemis. Mais reprenons le fil des événements. Il paraît que ma retraite à Montmorency déconcerta Mme d'Epinay ; vraisemblablement elle ne s'y était pas attendue. (Jean-Jacques Rousseau, Les Confessions, p. 586, Livre dixième ; 1778)

En (19) les troupes de Napoléon trouvent, en entrant dans une ville, des blessés qui ont péri dans un incendie :

3 Cela a été signalé également pour le verbe anglais seem par Aijmer 2009: 76. 
(19) Cet hôpital et ces maisons bordaient une large rue du côté de droite, en entrant. Il paraît que, au moment où l'incendie se manifesta, on sortit les blessés qu'on plaça au long des maisons, vis-à-vis de l'hôpital, en bordure de l'autre rive de la rue.

Sans doute, l'incendie fit de si rapides progrès que le rang des maisons où étaient adossés ces hommes prit feu. Les pans de bois brûlèrent ; des parties enflammées tombèrent sur ces malheureux. (Capitaine Gervais Souvenirs d'un soldat de l'Empire, p. 214 ; 1850)

Il s'agit ici d'un récit autobiographique. Il paraît que2 ne renvoie pas à un discours d'autrui. C'est bien une conjecture faite par le capitaine Gervais à partir de la scène qu'il a eue sous les yeux. Cela est confirmé par l'emploi de sans doute au paragraphe suivant.

(20) Quelques bourgeois nous faisaient signe que le pont était miné, mais nous étions déjà engagés dessus en poursuivant les Russes ; il paraît qu'il ne l'était pas, car il n'a pas sauté. (H. Scheltens, Souvenirs d'un grenadier de la garde, p. 114 ; c. 1860)

Il s'agit encore ici d'un emploi nettement inférentiel. Le fait que le pont n'a pas sauté est utilisé par le locuteur comme un indice qui lui permet de conclure à $\mathrm{p}$ " il n'était pas miné ». Le marqueur est proche ici de apparemment, ou même de devoir épistémique.

En (21), paraît que, relevant du français parlé familier, renvoie à une inférence établie subjectivement par le locuteur à partir des propos de son camarade. Le marqueur signifie «il semble que, on dirait que » :

(21) Tout à coup Picart s'arrêta en disant: " Coquin de Dieu! sentez-vous comme moi, mon pays, comme ce tartare a le trop dur? " Je lui répondis qu'il nous faisait souffrir par vengeance de ce que nous avions tué son maître: «Diable! me dit-il, paraît, mon sergent, que la petite goutte a fait son effet et que vous avez le petit mot pour rire!» (Sergent Bourgogne, Mémoires, p. 191 ; rédigés vers 1853)

Comme c'est le cas pour apparemment, le locuteur peut s'appuyer sur un discours d'autrui, utilisé comme un indice, pour conclure à $\mathrm{p}$ :

(22) On l'inonda d'ablutions, on le tourmenta de jeûnes et de prières, et l'on parvint, dit-on, à chasser le diable ; mais d'après ce qu'en rapportent des témoins éclairés, il paraît que ces possédés ne sont pas autre chose que des hommes frappés de folie, de manie et d'épilepsie ; et il est très-remarquable que le même mot arabe désigne à-la-fois l'épilepsie et l'obsession. (Comte de Volney, Voyage en Égypte et en Syrie, p. 29, chap. 24 ; 1787)

Il paraît que ne serait pas ici nécessaire, il sert à atténuer la prise en charge de l'assertion par le locuteur, avec un sens « il semble que ». Il paraît que2 inférentiel va subsister tout au long du XIX ${ }^{\mathrm{e}}$ siècle ; il était encore en usage au début du $\mathrm{xx}^{\mathrm{e}}$ siècle.

L'évolution de il paraît que à valeur inférentielle se fait en deux étapes. Dans une première étape, il paraît que1 a une valeur intersubjective. L'inférence qu'il réalise relève de la logique, d'un savoir général que tout le monde est censé partager. Dans une deuxième étape, il paraît que2 évolue vers une plus grande subjectivité ; il est alors en rapport avec le savoir du locuteur en particulier. La conclusion introduite par le marqueur a été inférée par lui à partir d'indices. Il paraît que suit là une tendance générale dans l'évolution des expressions modales épistémiques. Il se rapproche en ce sens de l'évolution de must, apparently, probably en anglais, telle qu'elle a été décrite dans Hanson (1987). 
Nous avons établi une évolution très similaire en deux étapes pour l'adverbe modal épistémique apparemment (voir Rodríguez Somolinos, 2010). Il paraît que, comme apparemment, a suivi ainsi le processus de subjectivisation signalé par Traugott (1989) pour les marqueurs épistémiques et médiatifs de l'anglais. Ils évoluent vers une plus grande prise en compte du savoir, des croyances ou de l'attitude du locuteur envers l'énoncé.

\section{IL PARAIT QUE3, MARQUEUR D'OUÏ-DIRE}

Dans le courant du $\mathrm{XIX}^{\mathrm{e}}$ siècle on voit apparaître un il paraît que3 renvoyant à un dire, à une information rapportée. Le premier exemple vraiment net date de 1826 :

(23) Avec un air si doux et si gentil, il paraît que c'est un diable, du moins à ce que m'a dit Madame Pinchon ; (Eugène Scribe, Le Mariage de raison, p. 385, Acte I, Scène I ; 1826)

(24) C'est aujourd'hui sa noce avec Déidamia. Quel bon cœur de quinze ans ! Et quelle ménagère ! S'il fut jamais aimé, c'est bien de celle-là. Un soldat m'a conté l'histoire de la bière. Il paraît que d'abord Frank s'était mis dedans. Deux de ses serviteurs, ses deux seuls confidents, fermèrent le couvercle, et, dès la nuit venue, le prêtre et les flambeaux traversèrent la rue. (Alfred de Musset, La Coupe et les lèvres, p. 325, Acte 5, Scène 2 ; 1832)

C'est là l'emploi moderne qui va coexister tout au long du XIX ${ }^{\mathrm{e}}$ siècle et jusqu'au début du $\mathrm{xx}^{\mathrm{e}}$ siècle avec il paraît que2, marqueur médiatif inférentiel. La transition de il paraît que2 inférentiel à il paraît que3, marqueur d'ouï-dire a pu se faire au début du XIX ${ }^{\mathrm{e}}$ siècle dans des contextes de ce type :

(25) J'ai vu ce matin Mr Didion frère et Emma. Ils sont arrivés à 10 h. du matin. J'étais au lit, et il a fallu me lever vite pour les recevoir. Ils se sont excusés de n'être pas venus à $8 \mathrm{~h}$. du matin. Il paraît que leur frère a compris ainsi et leur a écrit que je me levais avant le jour.

(George Sand, Correspondance, Lettre à Casimir Dudevant, p. 636 ; 1830)

Il est difficile de décider ici s'il s'agit d'un emploi inférentiel ou d'une information rapportée. Dans le premier cas, le locuteur déduit p à partir des propos des visiteurs avec un sens « il semble que »; c'est là l'interprétation la plus probable. Dans le deuxième cas, il reprend un dire d'autrui. Le lien entre ces deux types de médiativité est ténu et certains marqueurs cumulent les deux. C'est le cas par exemple de l'espagnol por lo visto et parece que (voir Cornillie, 2007), ainsi que de l'anglais apparently. Signalons qu'il s'agit de marqueurs qui ont un lien au départ avec la perception visuelle.

Dans le dernier quart du xix ${ }^{\mathrm{e}}$ siècle, l'emploi de il paraît que3 comme marqueur d'ouï-dire est bien établi :

(26) Bourdon : Il paraît que vous avez un faible pour cette jeune fille?

Teissier : Qui vous a dit cela?

Bourdon : Sa mère. (Henry Becque, Les Corbeaux, p. 186, Acte 3, Scène 7 ; 1882)

Ici il paraît que est interprété nécessairement comme renvoyant à un dire, puisque l'allocutaire enchaîne par Qui vous a dit cela ?

Il paraît que3 est compatible par ailleurs avec le conditionnel épistémique ou d'altérité énonciative, dont il est proche sémantiquement. Tous les deux sont polyphoniques et font allusion à un locuteur distinct (voir Haillet $2002: 14-16$ ) :

(27) La Comtesse de Foder 
Mais ce n'est pas tout, voici ce qu'on raconte. Il paraît que Paul Astier, sitôt les délais légaux, épouserait la demoiselle. (Alphonse Daudet, La Lutte pour la vie, p. 409 ; 1890)

Il paraît que2 inférentiel est toujours en usage vers la même époque :

(28) Lucienne : Eh bien ! moi, je ne sais pas votre avis là-dessus, mais il me semble que si j'étais homme, ce moyen de conquête ne serait pas de mon goût. Parce que, de deux choses l'une : ou la femme m'évincerait et je serais Gros-Jean comme devant ! pas la peine ! ou alors elle m'accueillerait et cela m'enlèverait du coup toute envie de la femme.

Pontagnac, embarrassé : Oui, évidemment !... (À part.) Ça va durer longtemps, ce marivaudage ? ...

Lucienne : Oui, mais il paraît que ce n'est pas l'avis de tous les hommes, si j'en juge par celui qui s'obstine à me suivre. (Georges Feydeau, Le Dindon, Acte I, Scène II, p. 36 ; 1896)

Le mouvement argumentatif est ici explicité par si j'en juge par. Lucienne tire ici une conclusion à partir de « quelqu'un s'obstine à me suivre ». Le marqueur est interprété comme « il semble que, apparemment».

Au début du $\mathrm{xx}^{\mathrm{e}}$ siècle, il paraît que3, marqueur médiatif d'ouï-dire, continue à alterner avec il paraît que2, marqueur médiatif inférentiel, même si la fréquence de celui-ci diminue jusqu'à sa disparition, qui date d'environ 1925. À partir de cette date, il paraît que est uniquement un marqueur médiatif d'ouï-dire. En ce sens il est polyphonique, il renvoie à une voix autre et correspond à une information rapportée. Il indique que le locuteur a acquis l'information soit à partir des propos d'autrui, soit à partir de rumeurs (voir Dendale et van Bogaert, 2007 : 76-77). La source de l'information n'est pas nécessairement explicitée. Selon la description que donne Haillet (2003 : 99), dans il paraît que p le locuteur n'assume pas le point de vue de p, qui est attribué à une instance distincte et mis à distance.

En français moderne, il paraît que3, marqueur d'ouï-dire, est figé. Il ne peut pas être nié et le verbe doit être au présent de l'indicatif. Il ne paraît pas que sera interprété comme «il ne semble pas que »:

(29) A ces questions, comme à d'autres semblables, il ne paraît pas que la science puisse encore définitivement répondre. (P. Theilhard de Chardin, Le Phénomène humain, p. 44 ; 1955)

Lorsque le verbe n'est pas au présent de l'indicatif, la structure impersonnelle ne correspond pas à un marqueur d'ouï-dire. Il paraissait que, à l'imparfait, est proche du verbe sembler :

(30) Mais bientôt, mon attention fut attirée vers les régions d'Amiens et de l'Oise où il paraissait que l'ennemi se préparait à l'offensive. (Joseph Joffre, Mémoires 1910-1917, $4^{\mathrm{e}}$ partie, Chap. 2, p. 199 ; 1931)

Il paraissait que renvoie uniquement à une information rapportée lorsqu'il introduit un discours indirect libre, auquel cas il équivaut à il paraît que3 :

(31) Ils entrèrent donc. Il était tard. Lily Brik, qui était venue de Moscou pour passer quelques jours de vacances automnales auprès de sa sœur Elsa et de son beau-frère Louis, était très déçue par l'atmosphère qui régnait dans les rues de Paris. Il paraissait que le cocktail à l'ambassade n'avait pas revêtu l'éclat qu'il avait connu les années précédentes. Beaucoup de personnes invitées avaient renoncé à venir sabler la vodka et manger le bon 
caviar qu'elles avaient appréciés en d'autres circonstances. Bref, le voyage de Lily à Paris était décevant. (Simone Signoret, La nostalgie n'est plus ce qu'elle était, p. 174 ; 1976) 


\title{
5. LES CONSTRUCTIONS EN INCISE :
}

\author{
A CE QU ’IL PARAIT, IL PARAIT, PARAIT-IL
}

À côté de la structure il paraît que $\mathrm{p}$, le français connaît depuis le XVIII ${ }^{\mathrm{e}}$ siècle plusieurs constructions en incise : à ce qu'il paraît, il paraît, comme il paraît, ou encore paraît-il, avec un sujet postposé. Ces constructions en incise interviennent au milieu ou à la fin d'un syntagme verbal. Elles portent sur l'ensemble de la proposition dans laquelle elles sont insérées (voir Willems et Blanche-Benveniste, $2010)^{4}$.

L'ancien français présentait déjà des constructions en incise. Nous avons vu en (2) si com il apert encore, qui a la même valeur sémantique que son équivalent il/si pert que.

Pour ce qui est du verbe paraître, l'incise la plus ancienne est à ce qu'il paraît, attestée dans Frantext dès 1755. Elle est aussi la plus fréquente de beaucoup, surtout au XIX ${ }^{\mathrm{e}}$ siècle :

(32) J'ai reçu d'Angleterre la réponse pour le vin que vous m'avez fait envoyer à milord Eliban ; il a été trouvé extrêmement bon. On me demande une commission pour quinze tonneaux, ce qui fera que je serai en état de finir ma maison rustique. Le succès que mon livre a eu dans ce pays-là contribue, à ce qu'il paraît, au succès de mon vin. (Montesquieu, Correspondance 1716-1755, p. $442 ; 1755)$

(33) Dîner avec la princesse de Bronsvic, sœur de l'impératrice de Russie, jolie personne, mais assez insignifiante, à ce qu'il paraît. Le soir les hussites de Kotzbue, détestable pièce. 60 enfants à la fois sur le théâtre. (Benjamin Constant, Journaux intimes, février 1804, p. 59 ; 1816)

Dans les deux cas, l'incise a un sens « semble-t-il », c'est-à-dire qu'elle équivaut sémantiquement à il paraît que2, en usage à cette époque. La proposition p sur laquelle porte à ce qu'il paraît correspond à une opinion personnelle du locuteur, à une conclusion à laquelle il est arrivé à partir d'indices indéterminés.

Les autres constructions en incise datent du XIX ${ }^{\mathrm{e}}$ siècle. Il paraît apparaît dans Frantext en 1840, comme il paraît en 1854. Elles présentent toutes les deux au départ un sens « semble-t-il » et correspondent à il paraît que2 :

(34) Théodore De Bèze était vieux alors et ne sortait guère de son logis ; François De Sales y vint incognito. Ils se donnèrent, il paraît, des marques d'estime mutuelles et même d'affection. C'étaient deux beaux-esprits, deux hommes modérés, d'un cœur fin et tendre. (Sainte-Beuve, Port-Royal, t. 1, p. $271 ; 1840$ )

(35) Quant au petit manuscrit que vous avez la bonté de réclamer, si je ne vous l'ai pas rendu, comme il paraît, je ne sais en vérité où le prendre. Dans le cas où je le retrouverais, je vous l'enverrai très certainement. (Félicité de Lamennais, Lettres inédites ... à la baronne Cottu (18181854), p. $130 ; 1854)$

Finalement, le français connaît à partir de 1859 une construction en incise paraît-il :

$4 \quad$ Willems et Blanche-Benveniste (2010) parlent de verbes faibles pour il me semble, il paraît, j'ai l'impression, on dirait, je dirais. Cela correspond à ce que Urmson (1952) appelle verbes parenthétiques. 
(36) - J'ignorais, il y a cinq minutes, que Mademoiselle de Beaupréau eût une dot, et je me trouvais assez riche pour elle et pour moi.

-Vraiment ? Dit M. de Kergaz. J'ai ouï dire le contraire. On m'a parlé même d'un homme portant un nom d'emprunt, chassé de Londres comme voleur et chef de bandits, qui était venu chercher fortune à Paris. Cet homme, paraît-il, avait eu connaissance du testament de M. de Kermarouët et il avait lentement ourdi une vaste intrigue dont je tiens à peu près tous les fils aujourd'hui. (P.-A. Ponson du Terrail, Rocambole, les drames de Paris, t. 1, p. 719 ; 1859)

Comme l'indique très nettement le contexte J'ai ouï dire le contraire. On m'a parlé même d'un homme ..., paraît-il est ici un marqueur d'ouï-dire équivalant à il paraît que3, qui est déjà en usage à cette époque.

Il faut ajouter à ces incises la construction disjointe il paraît, dans laquelle la structure impersonnelle fonctionne en emploi absolu; elle forme une réplique indépendante dans un tour de parole. « Le verbe faible fonctionne comme un noyau autonome » (Willems et Blanche-Benveniste, 2010 : 570-71). La première occurrence dans Frantext date de 1844 :

(37) - Sept mille livres en louis de douze francs.

- Sept mille livres! s'écria Porthos, ce mauvais petit diamant valait sept mille livres ?

-Il paraît, dit Athos, puisque les voilà ; je ne présume pas que notre ami d'Artagnan y ait mis

du sien. (Alexandre Dumas, Les Trois mousquetaires, p. 532 ; 1844)

Elle a une valeur inférentielle, au sens de « apparemment » ou « c'est ce qu'il semble ».

Comme le signalent Willems et Blanche-Benveniste (2010 : 571), en français moderne il paraît que p connaît la construction en incise - il paraît, paraît-il - et aussi la construction disjointe - il paraît, paraît. La valeur est toujours « source externe du savoir ", c'est-à-dire qu'il s'agit d'un marqueur médiatif d'information rapportée.

L'étude diachronique en profondeur des constructions en incise est encore à faire. En ce qui concerne à ce qu'il paraît, il paraît, paraît-il, etc.., nous nous limiterons à signaler que ces constructions sont très proches sémantiquement de il paraît que p. Les incises suivent en parallèle l'évolution de la structure introduisant une proposition subordonnée.

\section{CONCLUSION}

L'évolution de il paraît que s'est faite en trois étapes :

1. Il pert que, il appert que, il paroist que, il paraît que1 présentent un lien étroit avec la perception visuelle. En français médiéval et préclassique, ils ont le plus souvent une valeur médiative inférentielle, avec un sens « on voit bien que, il est évident que ». La structure logique de l'inférence par raisonnement est souvent explicitée par des structures d'où il appert que, parquoy il appert que, «d'où l'on peut déduire que, ce qui vient prouver que ». Le lien inférentiel mis en place par ces marqueurs se présente comme intersubjectif. Il relève d'un savoir général, indépendant de la subjectivité du locuteur. Il paraît que1, apparu vers 1650, est en usage jusqu'à la fin du XVIII ${ }^{\mathrm{e}}$ siècle.

2. En français classique se développe il paraît que2, qui a évolué vers une plus grande subjectivité. L'inférence repose sur le savoir du locuteur en particulier. Le marqueur indique que celui-ci a des raisons pour induire $\mathrm{p}$ à partir de certains indices, qui ne sont pas toujours 
repérables. Il paraît que2 est proche de il semble que et de apparemment dans leur emploi moderne. il disparaît au début du $\mathrm{xx}^{\mathrm{e}}$ siècle.

3. Il paraît que3 est un marqueur d'ouï-dire, il renvoie à une information rapportée. Apparu dans la langue dans le premier tiers du XIX ${ }^{\mathrm{e}}$ siècle, il devient le seul emploi possible à partir de 1925 environ.

Tout comme le conditionnel épistémique, le il paraît que3 moderne est un marqueur d'opération d'emprunt d'information (voir Dendale 1994 : 38). L'information est créée par autrui. D'autres marqueurs médiatifs réalisent une opération de création d'information. C'est le cas de devoir épistémique, de apparemment, de il m'est avis que ou encore de il paraît que2. Le locuteur crée luimême l'information et en est par conséquent responsable.

Il paraît que est passé en diachronie d'une catégorie à l'autre, d'une valeur inférentielle -création d'information- à une valeur d'ouï-dire -emprunt d'information-. Le lien entre ces deux emplois tient au fait que la source de l'information est indirecte dans les deux cas. Par ailleurs dans ces deux emplois le marqueur a une valeur modale faible. Alors que Il pert que, il appert que, il paroist que, il paraît que1 établissent un lien inférentiel à valeur intersubjective, les emplois 2 et 3 de il paraît que effectuent une atténuation, une mise à distance ; le locuteur ne prend pas entièrement en charge l'assertion sur laquelle porte il paraît que. Le marqueur a connu ainsi un affaiblissement en diachronie. Il a évolué vers une plus grande subjectivité et un degré moindre de certitude de la part du locuteur.

Amalia RoDRIGUEZ SOMOLINOS

Universidad Complutense de Madrid 


\section{BIBLIOGRAPHIE}

Dictionnaires et bases de données

BFM -Base de Français Médiéval [En ligne], Lyon, ENS de Lyon, Laboratoire ICAR, 2012, <http://bfm.enslyon.fr>

Corpus de la littérature médiévale en langue d'oül des origines à la fin du $\mathrm{xv}^{\mathrm{e}}$ siècle, Paris, Champion électronique, 2001.

Huguet, E., Dictionnaire de la langue française du xvi ${ }^{\mathrm{e}}$ siècle (1925-1967), Paris, Champion électronique, 2004.

Textes de français ancien (TFA) [en ligne], Laboratoire de Français Ancien, The ARTLF Project, Université d'Ottawa, <http://artfl-project.uchicago.edu>

Frantext, ATILF - CNRS / Université de Nancy 2 : 〈http : //www.frantext.fr>

\section{Études}

AIJMER, K., 2009, Seem and evidentiality, Functions of language 16, 1, p. 63-88.

CHAFE, W., 1986, "Evidentiality in English conversation and academic writing”, dans W. Chafe J. Nichols (eds) Evidentiality: The linguistic coding of epistemology, Norwood NJ, Ablex Publishing Corp., p. 261-272.

CORNILLIE, B., 2007, "The continuum between lexical and grammatical evidentiality: a functional analysis of Spanish parecer", Rivista di Linguistica, 19, 1, p. 109-128.

DendALE, P., 1994, « Devoir épistémique, marqueur modal ou évidentiel ? », Langue Française 102, p. 24-40.

DENDALE, P. et TASMOWSKI, L., 2001, "Introduction: evidentiality and related notions", Journal of Pragmatics 33,3 , p. 339-348.

Dendale, P. et VAN BOgaerT, J., 2007,“A semantic description of French lexical evidential markers and the classification of evidentials", Rivista di Linguistica 19, 1, p. 65-89.

Grossman, F. et Tutin, A., 2010, "Evidential Markers in French Scientific Writing: The Case of the French Verb voir", dans E. Smirnova, et G. Diewald (eds) Linguistic Realization of Evidentiality in European Languages, Berlin and new York, Mouton de Gruyter, p. 279-308.

HAILlET, P., 2002, Le conditionnel en français. Une approche polyphonique, Coll. L'essentiel français, Paris, Ophrys.

HaILlet, P., 2003, « Polyphonie et modalisation », dans A. Rodríguez Somolinos (éd) Des mots au discours : Etudes de linguistique française. Thélème, $\mathrm{n}^{\circ}$ spécial, Universidad Complutense de Madrid, p. 95-108.

HANSON, K., 1987, “On Subjectivity and the History of Epistemic Expressions in English”, Chicago Linguistic Society 23,1 , p. 133-147.

LANLY, A., 1971, Fiches de philologie française, Paris, Bordas.

NøLKE, H., 2001, « La dilution linguistique des responsabilités. Essai de description polyphonique des marqueurs évidentiels il semble que et il paraît que », dans Le regard du locuteur 2, Paris, Kimé, p. 15-34.

NUYTS, J., 2001, "Subjectivity as an evidential dimension in epistemic modal expressions", Journal of Pragmatics 33, 3, p. 383-400.

REY, A., 2004 [1992], Dictionnaire historique de la langue française, Paris, Dictionnaires Le Robert.

RODRIGUEZ SOMOLINOS, A., 2010, «L'évolution de apparemment en français : la formation d'un marqueur d'attitude énonciative », dans B. Combettes et al. (éds) Le changement en français. Études de linguistique diachronique, Berne, Peter Lang, p. 345-361.

RODRIGUEZ SOMOLINOS, A., 2014, «L'évolution en français de il m'est avis que/ ce m'est avis : médiativité, perception, inférence », dans Actes du Congrès Mondial de Linguistique Française 2014, Freie Universität Berlin, 19-23 Julio 2014, institut de Linguistique Française/ EDP Sciences, p. 1-12, CD-Rom.

THUILLIER, F., 2004, « Le verbe paraître : surgissement, manifestation, ouï-dire », Linx 50, p.5-32. 
TraugotT, E. C., 1989, “On the rise of epistemic meanings in English: an example of subjectification in semantic change”, Language 57, p. 33-65.

URMSON, J., 1952, "Parenthetical verbs", Mind 61, p. 480-496.

Willems, D. et BLANCHE-BENVENISTE, C., 2010, «Verbes ‘faibles’ et verbes à valeur épistémique en français parlé : il me semble, il paraît, j'ai l'impression, on dirait, je dirais », dans M. Iliescu, H. Siller-Runggaldier, et P. Danler (éds), Actes du XXVe Congrès International de Linguistique et de Philologie Romane (Innsbruck 2007), Berlin / New York, De Gruyter, tome IV, p. 565-576.

\section{Textes cités}

BeCQue, Henri, Les Corbeaux, dans Théâtre Complet, t. 2, Paris, Fasquelle, 1922.

Beaudouin de Sebourc, L. Crist (éd), 2 vols, Abbeville, SATF, 2002.

CALVIN, Jean, Institution de la religion chrestienne, J. D. Benoit (éd), Paris, J. Vrin, 1957.

Chretien de Troyes, Cligès, A. Micha (éd), Paris, Champion, 1957.

ChretiEn DE Troyes. Erec et Enide, M. Roques (éd), Paris, Champion, 1962.

Constant, Benjamin, Journaux intimes, A. Roulin et G. Roth (éds), Paris, Gallimard, 1952.

DAUDET, Alphonse, La Lutte pour la vie, Paris, Librairie de France, 1890.

DuMAS, Alexandre, Les Trois mousquetaires, Paris, Gallimard, 1996.

FEYDEAU, Georges, Le Dindon, Paris, Gallimard, 2001.

Floire et Blancheflor, M. Pelan éd., Paris, Ophrys, 1975.

GAUTIER D’ArRAS, Eracle, Guy Raynaud de Lage (éd), Paris, Champion, 1976.

GERVAIS, Souvenirs d'un soldat de l'empire. À la conquête de l'Europe, Paris, Bernard Giovanangeli, 2009.

JOFFRE, Joseph, Mémoires 1910-1917, Paris, Plon, 1932.

LAMENnAIS, Félicité de, Lettres inédites ... à la baronne Cottu, le Comte d'Haussonville (éd), Paris, Perrin et Cie, 1910

LAVArdin, Jacques de, La Célestine, D. L. Drysdall (éd), Londres, Tamesis Books, 1974.

LEGRAND, Jacques, Livre de bonnes meurs, E. Beltran (éd), Paris, Champion, 1988.

Miracle de l'abbeesse grosse, in Miracles de Nostre Dame par personnages, G. Paris et U. Robert (éds), Paris, Firmin Didot, 7 vol, 1876-1897.

Montesquieu, Correspondance 1716-1755, F. Gebelin et A. Morize (éds), Paris, Champion, 1914.

SAINTE-BeuVE, Charles Port-Royal, Paris, Hachette, 1860.

Musset, Alfred de, La Coupe et les lèvres, dans Euvres complètes, F. Baldensperger et R. Doré (éds), t. 1, Paris, Conard, 1923.

Ponson du Terrail, Pierre-Alexis, Rocambole, t. 1, C. A. Ciccione (éd), Monaco, Editions du Rocher, 1963.

La Queste del Saint Graal, A. Pauphilet (éd), Paris, Champion, 1949.

RousseaU, Jean-Jacques, Les Confessions, B. Gagnebin et M. Raymond (éds), Paris, Gallimard, 1997.

SEvigne, Marie de Rabutin-Chantal, Correspondance, t. 2 et 3, Paris, Gallimard, 1974 et 1978.

Scheltens, H. Souvenirs d'un grenadier de la garde. Paris, Bernard Giovanangeli, 2005.

SAND, George, Correspondance, G. Lubin éd., Paris, Garnier, t. 1, 1987. 
SCRIBE, Eugène, Le Mariage de raison, dans Euvres complètes, Paris, Furne, t. 4, 1845.

Bourgogne, Adrien, (2013) Mémoires, Paris, Arléa.

SignORET, Simone, La nostalgie n'est plus ce qu'elle était, Paris, Editions du Seuil, 1976.

Teilhard de Chardin, Pierre, Le Phénomène humain, Paris, Editions du Seuil, 1962.

Volney, Constantin-François de Chasseboeuf, Voyage en Égypte et en Syrie, Paris, Volland, 1787.

VOLTAIRE, Essay sur l'histoire générale et sur les moeurs et sur l'esprit des nations, Genève, Cramer, 1756.

Voyage de La Pérouse autour du monde, L.M.A. Milet-Mureau (éd), Paris, Impr. de la République.

WACE, Roman de Rou, A. J. Holden (éd), Paris, Champion, SATF, vol. 2, 1971. 